\title{
Parity is associated with an expanded macrophage population in the mammary gland
}

\author{
WEI ZHAO ${ }^{1}$, CLINTON J. GRUBBS ${ }^{2}$, RONALD K. MYERS ${ }^{3}$ and MARIT NILSEN-HAMILTON ${ }^{1}$ \\ ${ }^{1}$ Department of Biochemistry, Biophysics and Molecular Biology, Iowa State University, Ames, IA 50011; \\ ${ }^{2}$ Department of Surgery, University of Alabama, Birmingham, AL 35294; ${ }^{3}$ Department of \\ Veterinary Pathology, Iowa State University, Ames, IA 50011, USA
}

Received May 12,2010; Accepted June 28, 2010

DOI: 10.3892/ijo_00000771

\begin{abstract}
Pregnancy is a well established protective factor against breast cancer. One explanation for protection is the increased differentiation status of the parous epithelium. However, this does not explain the association of parity with increased aggressiveness of breast cancers, particularly cancers that occur soon after pregnancy. Because tumor aggressiveness can be influenced by the cell population that surrounds the mammary epithelium, we examined the potential role of the immune system in establishing a long-term difference between the mammary glands of primiparous and virgin animals. Specific mRNA levels, enzyme activities and antigen expressing cells were quantified in primiparous and virgin mammary glands from Sprague-Dawley rats in diestrous. Our results show that macrophages, but not neutrophils or B-cells, are specifically increased in fully involuted glands compared with age-matched virgin mammary glands. Macrophages play a dual role in tumor progression, both opposing and supporting the process. Our finding of an increased macrophage population in the primiparous mammary gland could explain the dichotomy of the reported association of parity with decreased breast cancer incidence and increased breast cancer aggressiveness.
\end{abstract}

\section{Introduction}

It is well established that parity conveys protection against cancers of the breast and other reproductive tissues. This correlation has been demonstrated in many studies using rodent models and from epidemiological analyses of human populations. Full-term pregnancy seems necessary for

Correspondence to: Dr Marit Nilsen-Hamilton, 3206 Molecular Biology Building, Department of Biochemistry, Biophysics and Molecular Biology, Iowa State University, Ames, Iowa 50011, USA

E-mail: marit@iastate.edu

Key words: parity, mammary gland, macrophage protection against breast cancer $(1,2)$. However, the age at first parity affects both the premenopausal and postmenopausal risk for breast cancer (3). A meta-analysis of the compiled data from 47 epidemiological studies in 30 countries, which included 50302 women with breast cancer and 96973 women without breast cancer, showed a $4.3 \%$ decrease in the relative incidence of breast cancer for every 12 months of breastfeeding in addition to a $7.0 \%$ decrease in breast cancer risk for each birth (4). The study also showed that protection from cancer obtained by breast feeding or pregnancy was the same in developed and undeveloped countries. The authors concluded that almost $70 \%$ of the increased protection against breast cancer observed in parous females of developing countries can be accounted for by the larger number of births per female and the increased frequency of breast feeding by these latter populations (4).

Although parity protects against the occurrence of breast cancer, it is also positively associated with increased aggressiveness of breast cancer and decreased cancer survival rates (5-9). Thus, the effect of reproductive status on breast cancer is complex, with full-term pregnancy and lactation contributing to decreased cancer incidence and increased tumor aggressiveness.

A change in the differentiation state of the mammary epithelium or another cell type has been suggested as the reason for parity-induced protection against tumor incidence (10) and increased p53 expression was proposed as the mediator of this effect (11). However, an increased differentiation status of the mammary epithelium does not explain the increased incidence of aggressive tumors in parous compared with nulliparous females.

Microarray analyses have identified a small group of genes that remain elevated in the fully involuted mouse and rat mammary gland $(12,13)$. Although the two studies did not produce the same list of upregulated genes, there was some overlap. First, the two studies were in agreement that expression of the major milk protein genes (a sign of epithelial cell differentiation) remained elevated after 26-28 days of involution. Second, both studies showed elevated expression of several genes that are expressed exclusively or highly by macrophages. Third, the expression of some genes associated with inflammation and the immune response were elevated in primiparous glands, particularly in the mouse study. The 
results of these studies suggest the interesting possibility that a consequence of parity is an increase in size of the immune cell population of the fully involuted mammary gland and that these immune cells influence epithelial differentiation status and tumor aggressiveness.

We evaluated the hypothesis that parity results in an increase in number of one or more hematopoietic cell type in the mammary gland using the diestrous rat as our model. Comparing the mammary tissue of primiparous and virgin animals we found, in the former, higher expression of genes that are upregulated during the innate immune response. We also found that primiparous glands contain a higher density of macrophages than the virgin glands. These results are consistent with the hypothesis that one contribution of parity to protection against breast cancer is a specific increase in the size of the mammary macrophage population in the involuted breast. The larger number of macrophages should provide more effective immune surveillance and decreased cancer incidence in parous compared with nulliparous females.

M1 macrophages, which protect against cancer, can be converted by tumor cells to M2 macrophages that are also referred to as tumor-associated macrophages (TAMs). Whereas M1 macrophages engulf tumor cells and protect against the development of cancer, M2 macrophages are believed to promote tumor progression by secreting growth factors and proteases, promoting angiogenesis and suppressing adaptive immunity (14). Because the parous mammary gland contains a larger pool of macrophages that are available for conversion to the M2 type it might be expected that cancers in the parous mammary gland are more frequently aggressive compared with those in the virgin breast. Thus, our observation of a specific increase in the macrophage population in parous mammary glands leads to an overarching hypothesis to explain the effect of parity on breast cancer that includes the influence of the macrophage on epithelial differentiation, immune surveillance and tumor aggressiveness.

\section{Materials and methods}

Experimental animals and materials. Female Sprague-Dawley rats were obtained from Harlan Company (Indianapolis, IN). The rats were allowed to undergo a 21-day lactation period after delivery. Rats were then allowed at least 28 days for complete involution of the mammary gland before being sacrificed. Virgin rats were sacrificed in the same week as the primiparous rats. All rats (primiparous and virgin) were sacrificed at approximately 135 days of age and on the second day of diestrous, which was confirmed by vaginal smear.

All animals were housed and treated according to current $\mathrm{NIH}$ guidelines. Animal care was provided by an animal caretaker and an attending veterinarian. This research was conducted in accordance with the standards set forth in the NIH guide for the care and use of laboratory animals. Animals were sacrificed with $\mathrm{CO}_{2}$ inhalation prior to removal of tissues for the described studies. Prior approval was obtained from the appropriate university Committee on Animal Care for all procedures performed on the animals used in these studies. Unless another source is identified, all chemicals used were reagent grade or higher and purchased from Sigma (St. Louis, MO) or Fisher (Fisher Scientific Inc. Fair Lawn, NJ).

RNA extraction and Northern blots. Tissues were frozen immediately in liquid nitrogen upon removal from the animal and stored at $-80^{\circ} \mathrm{C}$. Total RNA was isolated using TRIzol Reagent (Invitrogen, Carlsbad, CA) following the manufacturer's instructions. Northern blots were performed as previously described with $15 \mu \mathrm{g}$ total RNA per lane (15). The blots were hybridized with the following ${ }^{32} \mathrm{P}$-labeled probes produced by using the Megaprime DNA labeling system (Amersham Pharmacia Biotech, Little Chalfont, Buckinghamshire, England) with the following templates: Lcn2 (cDNA PCR product amplified by primers: AGTC GTCGACGACCTAGTAGCTGTGGAAACCATG and CCTAAAGCTTTCAGCCACACTCACCACCCA), SGP2 (EcoRI fragment from SGP-2 plasmid obtained from MD Griswold, Washington State University), GAPDH probe (XbaI-Pst I fragment of the rGAPDH plasmid obtained from JM Blanchard, Centre Paul Lamarque, France). Between hybridizations, the membranes were stripped by boiling 2 consecutive times in $0.1 \mathrm{X} \mathrm{SSC}$ with $0.1 \%$ SDS for $3 \mathrm{~min}$. Data were obtained by exposure to a phosphorimager (Molecular Dynamics, Sunnyvale, CA).

Quantitative RT-PCR. Reverse transcription was done as described previously (16). Briefly, $1 \mu \mathrm{g}$ total RNA was treated with 1 unit DNase (Invitrogen) for $15 \mathrm{~min}$. After de-activation of the DNase, the reverse transcription was performed using superscript II (Invitrogen) with the 18 mer oligo(dT) and dNTP. The qPCR was done in an Opticon (MJ Research, Waltham, MA) or a Mini Opticon (Bio-Rad Laboratories, Hercules, CA) with FullVelocity ${ }^{\mathrm{TM}}$ QPCR Master Mix (Stratagene, La Jolla, CA). The amount of each mRNA was quantified by using a standard curve created from a series of known dilutions of the amplified fragments. Values that were outside the range of the standards were not included and the affected samples were reanalyzed at dilutions to ensure that the values were within the range of the standard curve. The relative mRNA levels were calculated by normalizing the value for each mRNA to the result for the amount of the cyclophilin reference mRNA that had been quantified in the same sample. Primers used in the experiments were: Lcn2, sense: CAGGCCCAGGACTCAACTCAGAA, anti-sense: AGCGGCTTTGTCTTTCTTTCTGGA; cyclophilin: sense: TGTTCTTCGACATCACGGCTGAT, anti-sense: GGACTT GCCACCAGTGCCATTA; Igf1 sense: GGCACTCTGCTT GCTCACCTT, anti-sense: CGGAAGCAACACTCATCC ACA; Lbp sense: CTTGGCGTGGTCACGAATGTAT, antisense: GGAATGCCTGGAACAGGTTCA; SPP1 sense: ATG AGTCCTTCACTGCCAGCAC, anti-sense: TCATCGGACT CCTGGCTCTTC; Mmp12 sense: GGTCAAGATGGATGA AGCGGTAT, anti-sense: TCGTAATGTCAGCCTCGC CTT; Mpeg1 sense: AGTGATGGATGCCAAGTGTCCTA, anti-sense: TTGGTGGCAACTTGGCTCAT; TGFß3 sense: CTGTTGCGGAGAGAGTCCAACTT, anti-sense: GGTCA TCTTCGTTGTCCACTCCT; Emr1 sense: TTTCCTTGCCT GCTTCTTCT, anti-sense: CCTGTCTCCGTATTCAGCCA; CD14 sense: TACCGACCATGAAGCTTATGCT, antisense: GATTTGCTTCCGTGTCCACA; IL-6 sense: ACAG 
Table I. Expression of genes in rat mammary gland after weaning.

\begin{tabular}{llll}
\hline Gene & P/V & std & p-value \\
\hline${ }^{\Phi}$ Emrl & 2.79 & 2.54 & $0.003^{*}$ \\
SPP1 & 2.57 & 2.75 & $0.030^{*}$ \\
IL-6 & 2.07 & 1.61 & $0.015^{*}$ \\
${ }^{\Phi}$ Mmp12 & 2.05 & 1.70 & $0.027^{*}$ \\
${ }^{\Phi}$ Mpeg1 & 1.95 & 1.37 & $0.010^{*}$ \\
Lbp & 1.90 & 1.50 & $0.017^{*}$ \\
Lcn2 & 1.68 & 1.05 & $0.021^{*}$ \\
CD14 & 1.52 & 0.92 & $0.042^{*}$ \\
SGP-2 & 0.95 & 0.41 & 0.670 \\
IGF-I & 0.88 & 0.41 & 0.520 \\
TGFB3 & 0.52 & 0.62 & 0.150 \\
\hline
\end{tabular}

Total RNA from mammary glands of primiparous $(\mathrm{P})$ and age matched virgin $(\mathrm{V})$ rats were analyzed by Northern blot (SGP-2) and real-time RT-PCR (all others). mRNA of target genes were normalized to the reference gene, which was GPDH (Northern blots) or cyclophilin (real-time PCR) of the same sample. Fourteen parous rats and 9 virgin rats were used to obtain the data. Comparison was also made of the cyclophilin and GAPDH levels in parous $v$. virgin glands in each experiment dataset contributing to this table. In all cases there was no statistically significant difference between primiparous and virgin glands in the reference gene as determined by an unpaired Student's t-test. Genes expressed by macrophages are in italics. ${ }^{*} \mathrm{p}<0.05 ;{ }^{\Phi}$ macrophage specific genes.

\section{CCACTGCCTTCCCTACT, anti-sense: GAACTCCAGAAG ACCAGAGCAGA.}

Myeloperoxidase (MPO) assay. The MPO assay was performed according to the method of Bradley with minor modifications (17). Briefly, frozen tissues were weighed and homogenized in $1 \mathrm{ml}$ ice cold $0.5 \% \mathrm{HTAB}, 50 \mathrm{mM}$ potassium phosphate, $\mathrm{pH}$ 6.0. Homogenized samples were lysed by freeze-thaw and clarified by centrifugation. Samples $(20 \mu \mathrm{l})$ were assayed with an incubation time of 10 min followed by measuring the absorbance (A) at $450 \mathrm{~nm}$. The MPO activity was calculated with the formula: [( $\left.\left.\mathrm{A}^{10 \mathrm{~min}}-\mathrm{A}^{0 \mathrm{~min}}\right) \times 0.0565\right] /$ gram tissue $=\mathrm{MPO} /$ gram tissue (17) .

Immunohistochemistry. A polyclonal antibody recognizing the macrophage marker CD68 was used as the primary antibody (Santa Cruz Biotechnology, Santa Cruz, CA) for immunohistochemistry. Paraffin-embedded tissue sections from 6 mammary glands of primiparous rats and 8 mammary glands of virgin rats were deparaffinized and rehydrated. Antigen retrieval was done by boiling the sections then storing them at $95^{\circ} \mathrm{C}$ in $10 \mathrm{mM}$ Tris-EDTA, $\mathrm{pH}$ 9.0. Nova Red (Vector Laboratory, Burlingame, CA) was used for visualizing the streptavidin-biotin-conjugated horseradish peroxidase. Sections were then counterstained with Shandon hematoxylin (Fisher Scientific, Itasca, IL) to identify nuclei. Sectioning and staining was done by the Veterinary Pathology Services, Iowa State University.

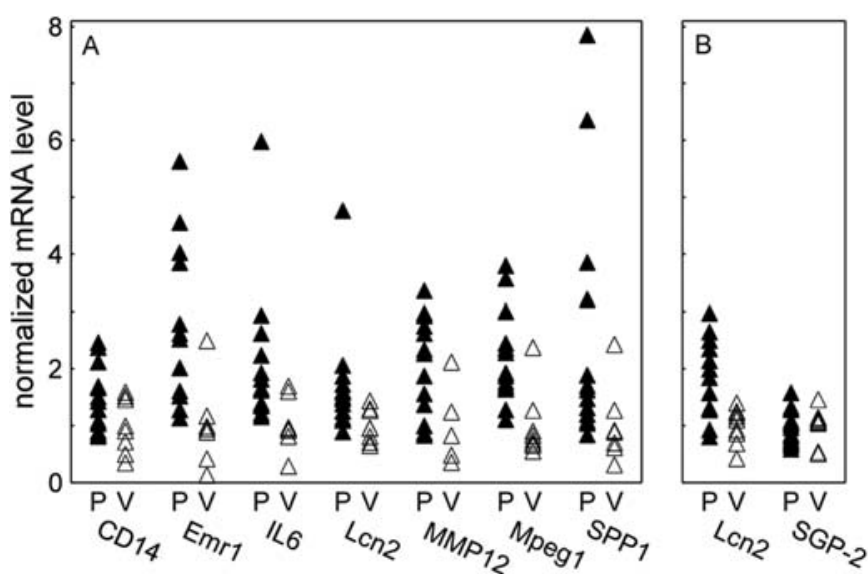

Figure 1. Macrophage gene expression is higher in primiparous mammary glands compared with in age-matched virgin glands. Total RNAs from mammary glands of rats were analyzed by (A) qPCR for which the values for the target mRNAs were normalized to the reference gene cyclophilin of the same sample, or (B) Northern blot, for which that values for the target mRNAs were normalized to the reference gene GPDH of the same sample. Samples from 14 primiparous rats and 9 virgin rats were used to obtain the data. In all cases there was no statistically significant difference between primiparous and virgin glands in the reference gene. $\mathrm{P}$, primiparous; $\mathrm{V}$, age matched virgin.

Macrophages were counted in ten randomly selected fields in each section, one section from each tissue sample. The counting was done blind with the identity of each sample only uncovered after the data had been collected. The total number of macrophages from the ten fields of each section was used as the data point for that tissue sample.

Statistics. The standard deviation for the quotient of the primiparous and virgin groups was calculated based on the standard deviation of each group (18). P-values were calculated by t-test.

\section{Results}

Effects of parity on the expression of immune responseassociated genes by the mammary gland. A previous microarray comparison of the genes differentially expressed in mammary glands of primiparous compared with virgin mice identified a set of genes associated with macrophages that were elevated in the primiparous mammary gland compared with age-matched virgin mammary gland (12). However, the tissues pooled for this study were not of a defined stage in estrous and were done with the mouse, which has not been commonly used as a model for parity-induced changes in mammary gland tissue.

We investigated the differences in gene expression between mammary glands of primiparous and virgin rats in diestrous with an emphasis on genes expressed by macrophages (Table I). Many of the mRNAs tested in the rat tissues were the same as those identified by the previous microarray of mouse tissues. However, some additional mRNAs that are selectively expressed by macrophages were also investigated. The results showed that the levels of several mRNAs that are expressed by macrophages were significantly elevated in the primiparous mammary glands. These mRNAs included CD14 


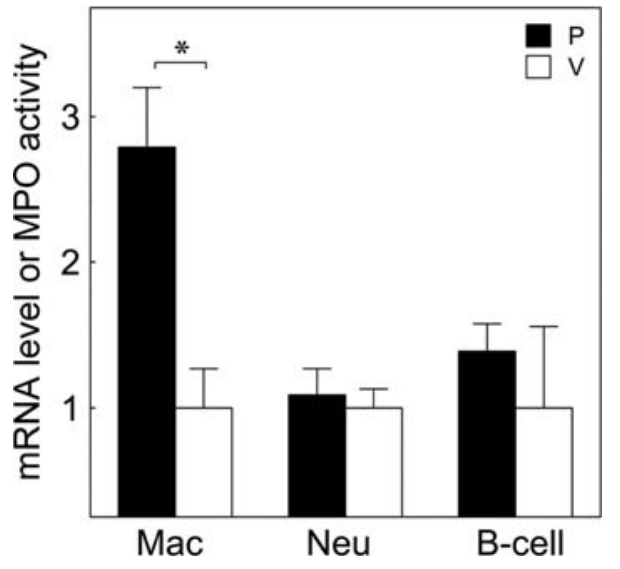

Figure 2. Macrophage-specific elevation of hematopoietic cell markers in primiparous mammary glands. Representative markers were tested to determine the relative population densities of immune cells in the mammary glands of primiparous rats and virgin rats. Real-time RT-PCR was used to detect macrophage marker Emr1 and B cell marker B220. The results were normalized to the level of cyclophilin in the same sample. Neutrophils were detected by the MPO assay. The Emr1 data are the same dataset as summarized for this mRNA in Fig. 1. The error bars show the standard error of the mean. ${ }^{*} \mathrm{p}<0.05$ as determined by an unpaired Student's t-test. Mac, macrophage; Neu, neutrophil; $\mathrm{P}$, primiparous; V, age-matched virgin.

antigen (CD14), interleukin-6 (IL-6), osteopontin (Spp1/Eta-1), macrophage-specific matrix metalloproteinase (MMP)-12, and macrophage expressed gene 1 (Mpeg1) (Fig. 1). We also found higher expression of Lbp in primiparous mammary gland, but we did not find significant differences in the mRNAs levels for IGF-1, TGFB3 and SGP2, all of which were previously reported to differ between the mouse primiparous and virgin tissues. Thus, the consistent finding between this study in the rat and the previous microarray study of the mouse mammary gland was that many macrophage markers increase with parity.

Macrophage markers, but not neutrophil or B-cell markers, are elevated in primiparous compared with virgin mammary glands. The population sizes of immune cells (neutrophils, macrophage, $\mathrm{B}$ and $\mathrm{T}$ cells) increase in the early stages of mammary gland involution (19-22). Our results from gene expression studies that suggest an increase in the macrophage population in the primiparous mammary gland might reflect the retention of a certain proportion of all immune cells after involution is completed. To determine which immune cell types are selectively retained in the primiparous mammary gland after the gland has completely involuted, we tested for these cell populations using MPO activity (neutrophils), and the gene markers Emr1 (macrophages), B220 (B cells) and OX40 (T cells) $(20,23,24)$. The result showed no differences in the neutrophil and B cell markers between primiparous and virgin mammary glands (Fig. 2). The expression of OX40 was not detectable in any sample (data not shown). By contrast the expression of the macrophage marker, Emr1, was higher in the primiparous compared to the virgin mammary glands $(\mathrm{p}<0.01)$.

Macrophages are more numerous in primiparous compared with age-matched virgin mammary glands. The observation
A
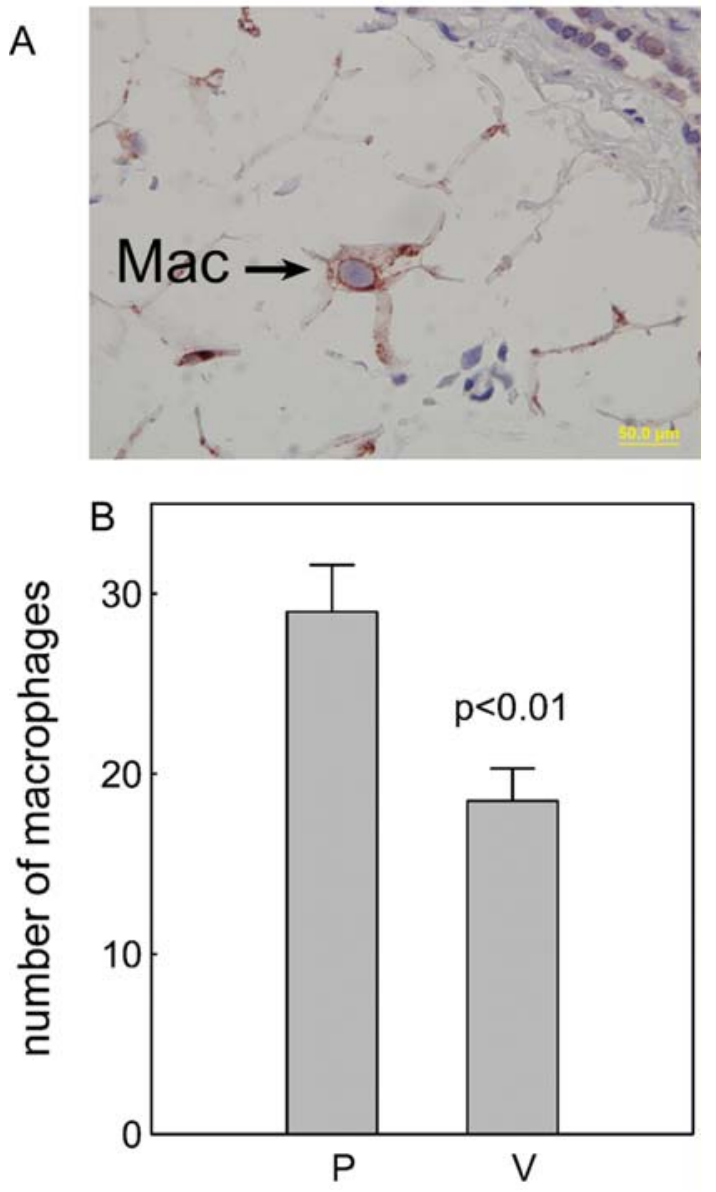

Figure 3. More macrophages are found in primiparous compared with virgin mammary glands. (A) Immunohistochemical staining with anti-CD68 of a paraffin-embedded tissue section from a rat mammary gland with x 1000 magnification. The yellow scaling ruler, inserted by the microscope in the bottom right corner, at the time of photography is $50 \mu \mathrm{m}$ in length. (B) Macrophages were counted from 10 randomly selected fields with x400 magnification of each section. The total number of macrophages in the ten fields was used for analysis. Error bars are the standard error of the mean from 6 primiparous samples and 8 virgin samples, respectively.

of elevated levels in primiparous glands of mRNAs that define macrophages could be explained by an increase in expression of these genes per cell or by an increase in the number of cells in the tissue. To determine if the macrophage population in primiparous glands is higher than in virgin glands we performed immunohistochemistry to detect and quantify macrophages. The results demonstrated that there were more macrophages in the fully involuted mammary glands than in the age-matched virgin glands $(\mathrm{p}<0.01)$ (Fig. 3).

Parity-induced changes in gene expression are specific for the mammary gland. To examine the possibility that the parity-induced changes in abundance of macrophage markers in the mammary gland reflects a body-wide change in macrophage content, we examined other tissues for similar differences in level of gene expression. For these studies the Lcn2 and Emr1 were chosen as the test genes and normalized to cyclophilin to examine specific changes in gene expression. As seen in Fig. 4, the difference between primiparous and virgin tissues in expression of Lcn2 and Emr1 was only 


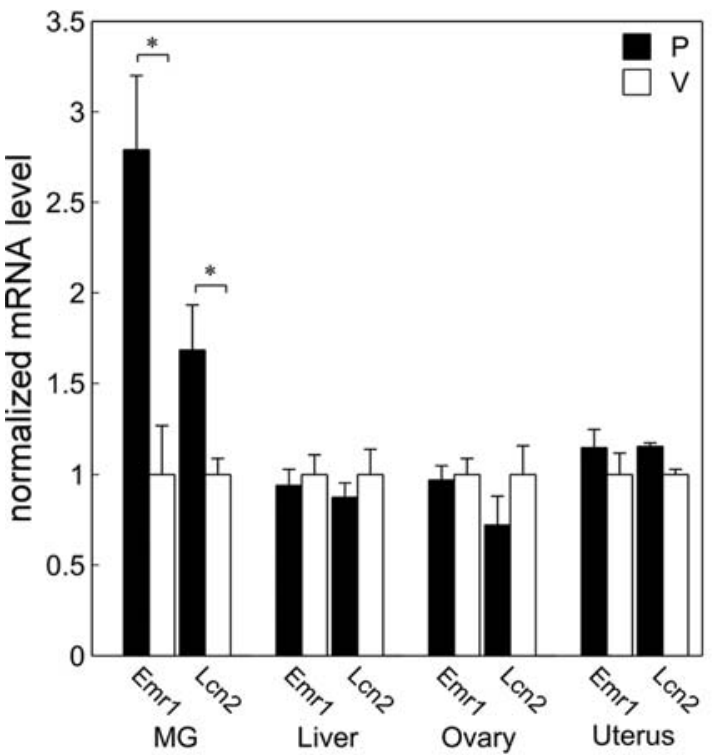

Figure 4. Selective increase in macrophage gene expression in mammary glands of the primiparous rat. Total RNA from liver, uterus, ovary and mammary gland of rats were analyzed by real-time RT-PCR for Emr1 and Lcn2. The values are the Emr1 or Lcn2 mRNA normalized to cyclophilin mRNA of the same sample. The Emr1 and Lcn2 data for the mammary gland are the same dataset as shown for these mRNAs in Fig. 1. P, primiparous; V, age matched virgin. Fourteen primiparous and 9 virgin rats were used in the experiment. Error bars show the standard error of the mean ${ }^{*} \mathrm{p}<0.05$.

observed in the mammary gland, thus showing that the effect of parity is specific for this gland.

\section{Discussion}

The participation of inflammatory cells in mammary gland involution has been reported in several studies in which the results of microarray analysis and histochemical staining indicated growth of the hematopoietic cell population, particularly neutrophils and macrophages, in the mammary gland during involution $(22,25,26)$. However, all these studies were of mouse mammary glands at early stages of involution (up to 4 days of involution).

Fully involuted mouse mammary glands showed higher levels of gene expression markers for macrophages, B-lymphocytes and T-lymphocytes in the involuted compared with the virgin glands (12). We also observed a difference in expression of macrophage marker genes (Mpeg1, Mmp12) between primiparous and virgin rat mammary glands, but did not observe evidence of significant differences in B-cell (B220) or neutrophil (MPO activity) populations. The difference in expression of Lbp, Mmp12 and Spp1 between primiparous and virgin glands was also observed in a recent microarray study of rat mammary glands (13).

A consistent finding in our study and the microarray analysis was of significant increases in the levels of expression of a number of markers for macrophages. From these results we reasoned that a possible explanation for the protective effect of parity against cancer is increased immune surveillance in the parous breast. Therefore, we examined the relative levels of markers for hematopoietic cells in involuted and virgin mammary glands. Neutrophil populations in the tissues were determined by the MPO assay $(17,27)$ and showed no difference between the primiparous and virgin groups. Analysis by real-time RT-PCR of B220, a B-lymphocyte marker, also demonstrated no difference between the two groups. Although microarray analysis found higher levels of immunoglobulin gene expression after involution compared with virgin mice $(12,25)$ the results of immunohistochemical staining of a B-cell marker showed no obvious changes in the B-cell number even in mammary glands at early stages of involution (25). Taken together, these results suggest that although the number of B-cells does not increase in the mammary gland after involution is completed, some immunoglobulin genes remain more active than in the virgin gland.

We tested the levels of several mRNAs, namely IGF-I, Lbp, Mmp12, Spp1, TGFB3 and SGP2, that showed altered expression between involuted and virgin mammary glands of mouse (12). Among them, Lbp, Mmp12 and Spp1 also showed higher expression in a microarray analysis of primiparous mammary glands of the rat (13). We also found higher expression of Mmp12 and Spp1 in the fully involuted rat mammary gland. We did not find differences in expression levels of IGF-I, TGFB3 and SGP2 and these latter genes also did not appear as up- or down-regulated in the rat microarray study although they did differ between primiparous and virgin gland in the mouse microarray. The difference in results for these mRNAs could be due to the species difference or to the immune status or other condition of the animals on sacrifice.

The expression levels of several macrophage markers [Mpeg1, Mmp12, CD14 and Emr1 (F4/80)] were higher in the mammary glands of primiparous compared with virgin rats. The mouse microarray study identified Mmp12 and Mpeg1 (12) and the rat study identified Mmp12 (13). To evaluate the macrophage population in the mammary gland, we stained tissue sections for the macrophage marker, CD68, and discovered that the macrophage density of the primiparous gland is higher than in the virgin mammary gland. Although not supported by earlier studies $(28,29)$ more recent studies have shown that the number of macrophages in the mammary gland increases during involution $(25,30,31)$. Thus, macrophages may enter the mammary gland during involution and a subset remains in the gland for at least 28 days when the rat mammary gland is considered to be fully involuted.

While parity protects against the occurrence of breast cancer, it is also positively associated with increased aggressiveness of cancer and decreased survival rates (5-9). These studies showed a large increase in cancer aggressiveness in breast cancer of parous compared with nulliparous females within $\sim 6$ years of parturition. Although most studies did not show a measurable effect of parity on tumor aggressiveness later in life, at least one study found an association of axillary lymph node involvement with parity in women with 4 pregnancies or more and this association was strongest with older women (7).

The association of parity with increased aggressiveness of breast cancer could be explained by the presence of larger numbers of macrophages in the mammary gland after parturition because macrophages are capable of being converted 
from the protective M1 macrophage to the tumor promoting M2 macrophage. Macrophages remaining in the mammary gland after pregnancy would contribute to creating a different microenvironment in the tissue than is present in the virgin gland. It has been argued that the reason for a higher frequency of more aggressive cancers after pregnancy is that a wound healing and inflammatory microenvironment is established in the mammary gland during involution and that this microenvironment is conducive to the proliferation of tumor cells (32). The macrophage is a component of both wound healing and inflammatory microenvironments and here we show that it is the one immune cell type that is elevated in primiparous compared with age-matched virgin rats.

Studies of the effects of hormonal treatments on the susceptibility to breast cancer have lead to proposals that the extent of differentiation of the epithelium in the parous breasts is greater than in virgin breasts (10). This proposal was supported by the finding that p53 is required to observe the effect of parity on carcinogen-induced mammary gland cancer $(33,34)$. However, p53 was not amongst the genes found to be differentially expressed in the primiparous compared with the virgin mammary gland $(12,13)$. Our finding of an increased number of macrophages in the primiparous gland is compatible with the previous hypothesis that epithelial differentiation is more complete in the primiparous gland and that $\mathrm{p} 53$ is required for differentiation to occur. However, we propose that the driving force that protects against cancer is the increased size of the mammary macrophage population. Macrophages are known to be associated with the terminal end buds and to promote differentiation of the mammary epithelium $(35,36)$.

During mammary gland involution, a local inflammatory response occurs in the absence of infection $(25,37)$. Lcn2 is an acute phase protein (APP) with high expression in the mammary gland and uterus during involution (15). Its expression level in the fully involuted mammary gland of primiparous rats is higher than in virgin rats. Lcn 2 is expressed by epithelial and hematopoietic cell types and induces apoptosis in neutrophils but not in macrophages (38). The persistently high expression of Lcn 2 in involuted mammary gland may be a reason that more macrophages but not neutrophils remain after involution is complete. To test this hypothesis, we compared the relative expression of macrophage marker, Emr1, and Lcn2 in a variety of tissues of primiparous and virgin animals and found that the expression of Emr1 is higher in primiparous compared with virgin mammary glands, but not in other reproductive tissues nor in liver. Lcn2 is highly expressed in these tissues on toxic or endotoxin challenge (liver) or during involution (uterus) or throughout the ovarian cycle (ovary). The similar proportional expression patterns of Emr1 and Len2 in primiparous compared with virgin animals suggests a relationship but our studies do not reveal more.

The increased Lcn2 might also reflect an increased inflammatory microenvironment in the primiparous gland. This was indicated by the increase in other acute phase proteins, particularly in the mouse microarray study. An increase in the expression of acute phase genes might also reflect a low level of inflammation in the glands (or one of the glands pooled for the microarrays) that is not associated with parity. In our study this was not so. Whereas the level of Lcn2 expression was higher in primiparous compared with virgin mammary glands, the level of SGP-2, another acute phase gene, was not different.

Parity also provides protection against endometrial cancer and ovarian cancer in humans as it does for breast cancer (39-45). We did not find evidence of more macrophages in the rat uterus and ovary of primiparous compared with virgin rats. This may imply that increased immune surveillance provided by macrophages is not a universal protective factor against cancer in different organs after parity and that it may not be the only mechanism for protection. However, the lack of evidence of protection against endometrial cancer and ovarian cancer provided by parity in rats makes it difficult to relate our results for these tissues to the results of studies of endometrial and ovarian cancers in women.

Decreased Lcn2 expression has recently been reported to suppress the formation of mammary gland cancer and its metastasis in Lcn2-/- MMTV-Erb2(V664E) and MMTV-PyMT transgenic mammary tumor mouse models $(46,47)$. Our results show a positive correlation between Lcn2 and macrophage markers in the primiparous gland, which could indicate that Lcn2 is involved in recruiting macrophages into the mammary gland. Decreased macrophage recruitment to the mammary gland results in decreased tumorigenesis and metastasis in the MMTV-PyMT mouse model (48-50). Lcn2 can also attenuate LPS-induced cytokine production in macrophages (51), the latter being a classical response associated with M1 macrophages (52). This action of Lcn2 would move macrophages to the M2 phenotype and thus Lcn2 may facilitate the conversion of macrophages from M1 to the M2 type that promotes mammary gland tumorigenesis and metastasis. Unlike the normal animal, the MMTV-PyMT and MMTVErb2(V664E) mouse model contains cells in the mammary gland that are predisposed to transformation. Thus, a change in the balance of M1 to M2 macrophages might have a proportionately larger effect on the expansion of cancer in these glands compared with in the mammary glands in a normal animal.

In summary, we have found that the number of macrophages is increased in involuted primiparous rat mammary glands compared to age-matched virgin glands. We propose that the resulting increased immune surveillance in the mammary gland may play a role in the protective effect of parity against breast cancer. Ironically, the higher frequency of macrophages in the primiparous gland may also be responsible for the observed positive association of parity and more aggressive breast cancers.

\section{Acknowledgements}

This work was supported in part by funds from the Agricultural Experiment Station, Ames, IA. The authors thank Lee Bendickson for helpful technical advice.

\section{References}

1. Newcomb PA, Storer BE, Longnecker MP, et al: Lactation and a reduced risk of premenopausal breast cancer. N Engl J Med 330: 81-87, 1994.

2. Russo J and Russo IH: Influence of differentiation and cell kinetics on the susceptibility of the rat mammary gland to carcinogenesis. Cancer Res 40: 2677-2687, 1980. 
3. Clavel-Chapelon F: Differential effects of reproductive factors on the risk of pre- and postmenopausal breast cancer. Results from a large cohort of French women. Br J Cancer 86: 723-727, 2002.

4. Collaborative Group on Hormonal Factors in Breast Cancer, Breast Cancer and Breast Feeding: collaborative reanalysis of individual data from 47 epidemiological studies in 30 countries, including 50302 women with breast cancer and 96973 women without the disease. Lancet 360: 187-195, 2002.

5. Colditz GA and Rosner BA: What can be learnt from models of incidence rates? Breast Cancer Res 8: 208, 2006.

6. Albrektsen G, Heuch I, Thoresen S and Kvale G: Clinical stage of breast cancer by parity, age at birth, and time since birth: a progressive effect of pregnancy hormones? Cancer Epidemiol Biomarkers Prev 15: 65-69, 2006.

7. Manjer J, Balldin G, Zackrisson S and Garne JP: Parity in relation to risk of axillary lymph node involvement in women with breast cancer. Results from Swedish population-based series of 3,472 consecutive cases. Eur Surg Res 37: 179-184, 2005.

8. Largent JA, Ziogas A and Anton-Culver H: Effect of reproductive factors on stage, grade and hormone receptor status in early-onset breast cancer. Breast Cancer Res 7: R541-R554, 2005.

9. Whiteman MK, Hillis SD, Curtis KM, McDonald JA, Wingo PA and Marchbanks PA: Reproductive history and mortality after breast cancer diagnosis. Obstet Gynecol 104: 146-154, 2004.

10. Russo J and Russo IH: Differentiation and breast cancer. Medicina (B Aires) 57 (Suppl. 2): S81-S91, 1997.

11. O'Malley CJ, McColl BK, Kong AM, Ellis SL, Wijayaratnam AP Sambrook J and Mitchell CA: Mammalian inositol polyphosphate 5-phosphatase II can compensate for the absence of all three yeast Sac1-like-domain-containing 5-phosphatases. Biochem J 355: 805-817, 2001.

12. D'Cruz CM, Moody SE, Master SR, et al: Persistent parityinduced changes in growth factors, TGF-beta3, and differentiation in the rodent mammary gland. Mol Endocrinol 16 : 2034-2051, 2002.

13. Uehara N, Unami A, Kiyozuka Y, Shikata N, Oishi Y and Tsubura A: Parous mammary glands exhibit distinct alterations in gene expression and proliferation responsiveness to carcinogenic stimuli in Lewis rats. Oncol Rep 15: 903-911, 2006.

14. Sica A, Larghi P, Mancino A, et al: Macrophage polarization in tumour progression. Semin Cancer Biol 18: 349-355, 2008.

15. Ryon J, Bendickson L and Nilsen-Hamilton M: High expression in involuting reproductive tissues of uterocalin/24p3, a lipocalin and acute phase protein. Biochem J 367: 271-277, 2002.

16. Fassett JT, Hamilton RT and Nilsen-Hamilton M: Mrp4, a new mitogen-regulated protein/proliferin gene; unique in this gene family for its expression in the adult mouse tail and ear. Endocrinology 141: 1863-1871, 2000.

17. Bradley PP, Priebat DA, Christensen RD and Rothstein G: Measurement of cutaneous inflammation: estimation of neutrophil content with an enzyme marker. J Invest Dermatol 78: 206-209, 1982

18. Robyt JF and White BJ: Biochemical Techniques Theory and Practice. Brooks/Cole Publishing Company, Monterey, CA, USA, 1987

19. Stein T, Salomonis N and Gusterson B: Mammary gland involution as a multi-step process. J Mammary Gland Biol Neoplasia 12: 25-35, 2007.

20. Atabai K, Sheppard D and Werb Z: Roles of the innate immune system in mammary gland remodeling during involution. J Mammary Gland Biol Neoplasia 12: 37-45, 2007.

21. Clarkson RWE, Boland MP, Kritikou EA, Lee JM, Freeman TC, Tiffen PG and Watson CJ: The genes induced by signal transducer and activators of transcription (STAT)3 and STAT5 in mammary epithelial cells define the roles of these STATs in mammary development. Mol Endocrinol 20: 675-685, 2006.

22. Clarkson RW, Wayland MT, Lee J, Freeman T and Watson CJ: Gene expression profiling of mammary gland development reveals putative roles for death receptors and immune mediators in post-lactational regression. Breast Cancer Res 6: R92-R109, 2004.

23. Gough MJ, Ruby CE, Redmond WL, Dhungel B, Brown A and Weinberg AD: OX40 agonist therapy enhances CD8 infiltration and decreases immune suppression in the tumor. Cancer Res 68 : 5206-5215, 2008.

24. Ohshima Y, Yang LP, Uchiyama T, et al: OX40 costimulation enhances interleukin-4 (IL-4) expression at priming and promotes the differentiation of naive human CD4(+) T cells into high IL-4-producing effectors. Blood 92: 3338-3345, 1998
25. Stein T, Morris J, Davies C, et al: Involution of the mouse mammary gland is associated with an immune cascade and an acute-phase response, involving LBP, CD14 and STAT3. Breast Cancer Res 6: R75-R91, 2004.

26. Monks J, Geske FJ, Lehman L and Fadok VA: Do inflammatory cells participate in mammary gland involution? J Mammary Gland Biol Neoplasia 7: 163-176, 2002.

27. Schneider T and Issekutz AC: Quantitation of eosinophil and neutrophil infiltration into rat lung by specific assays for eosinophil peroxidase and myeloperoxidase. Application in a Brown Norway rat model of allergic pulmonary inflammation. J Immunol Methods 198: 1-14, 1996.

28. Richards RC and Benson GK: Ultrastructural changes accompanying involution of the mammary gland in the albino rat. $J$ Endocrinol 51: 127-135, 1971 .

29. Mayberry HE: Macrophages in post-secretory mammary involution in mice. Anat Rec 149: 99-111, 1964

30. Atabai K, Fernandez R, Huang X, et al: Mfge8 is critical for mammary gland remodeling during involution. Mol Biol Cell 16: 5528-5537, 2005

31. Lund LR, Romer J, Thomasset N, et al: Two distinct phases of apoptosis in mammary gland involution: proteinaseindependent and -dependent pathways. Development 122: 181$193,1996$.

32. Schedin P, O'Brien J, Rudolph M, Stein T and Borges V: Microenvironment of the involuting mammary gland mediates mammary cancer progression. J Mammary Gland Biol Neoplasia 12: 71-82, 2007.

33. Medina D and Kittrell FS: p53 function is required for hormonemediated protection of mouse mammary tumorigenesis. Cancer Res 63: 6140-6143, 2003.

34. Sivaraman L, Conneely OM, Medina D and O'Malley BW: p53 is a potential mediator of pregnancy and hormone-induced resistance to mammary carcinogenesis. Proc Natl Acad Sci USA 98: 12379-12384, 2001.

35. Ingman WV, Wyckoff J, Gouon-Evans V, Condeelis $\mathrm{J}$ and Pollard JW: Macrophages promote collagen fibrillogenesis around terminal end buds of the developing mammary gland. Dev Dyn 235: 3222-3229, 2006.

36. Gouon-Evans V, Rothenberg ME and Pollard JW: Postnatal mammary gland development requires macrophages and eosinophils. Development 127: 2269-2282, 2000.

37. Nilsen-Hamilton M, Liu Q, Ryon J, Bendickson L, Lepont P and Chang Q: Tissue involution and the acute phase response. Ann NY Acad Sci 995: 94-108, 2003.

38. Devireddy LR, Teodoro JG, Richard FA and Green MR: Induction of apoptosis by a secreted lipocalin that is transcriptionally regulated by IL-3 deprivation. Science 293: 829834, 2001

39. Gierach GL, Modugno F and Ness RB: Relations of gestational length and timing and type of incomplete pregnancy to ovarian cancer risk. Am J Epidemiol 161: 452-461, 2005.

40. Hanna L and Adams M: Prevention of ovarian cancer. Best Pract Res Clin Obstet Gynaecol 20: 339-362, 2006.

41. Lambe M, Wuu J, Weiderpass E and Hsieh CC: Childbearing at older age and endometrial cancer risk (Sweden). Cancer Causes Control 10: 43-49, 1999.

42. Modugno F, Ness RB, Chen C and Weiss NS: Inflammation and endometrial cancer: a hypothesis. Cancer Epidemiol Biomarkers Prev 14: 2840-2847, 2005

43. Pettersson B, Adami HO, Bergstrom R and Johansson ED: Menstruation span-a time-limited risk factor for endometrial carcinoma. Acta Obstet Gynecol Scand 65: 247-255, 1986.

44. Pike MC, Pearce CL and Wu AH: Prevention of cancers of the breast, endometrium and ovary. Oncogene 23: 6379-6391, 2004.

45. Purdie DM and Green AC: Epidemiology of endometrial cancer. Best Pract Res Clin Obstet Gynaecol 15: 341-354, 2001.

46. Berger T, Cheung CC, Elia AJ and Mak TW: Disruption of the Lcn 2 gene in mice suppresses primary mammary tumor formation but does not decrease lung metastasis. Proc Natl Acad Sci USA 107: 2995-3000, 2010.

47. Leng X, Ding T, Lin H, et al: Inhibition of lipocalin 2 impairs breast tumorigenesis and metastasis. Cancer Res 69: 8579-8584, 2009.

48. Aharinejad S, Paulus P, Sioud M, et al: Colony-stimulating factor- 1 blockade by antisense oligonucleotides and small interfering RNAs suppresses growth of human mammary tumor xenografts in mice. Cancer Res 64: 5378-5384, 2004. 
49. Lin EY, Nguyen AV, Russell RG and Pollard JW: Colonystimulating factor 1 promotes progression of mammary tumors to malignancy. J Exp Med 193: 727-740, 2001.

50. Paulus P, Stanley ER, Schafer R, Abraham D and Aharinejad S: Colony-stimulating factor- 1 antibody reverses chemoresistance in human MCF-7 breast cancer xenografts. Cancer Res 66: 4349-4356, 2006.
51. Zhang J, Wu Y, Zhang Y, Leroith D, Bernlohr DA and Chen X: The role of lipocalin 2 in the regulation of inflammation in adipocytes and macrophages. Mol Endocrinol 22: 1416-1426, 2008.

52. O'Brien J and Schedin P: Macrophages in breast cancer: do involution macrophages account for the poor prognosis of pregnancy-associated breast cancer? J Mammary Gland Biol Neoplasia 14: 145-157, 2009. 